\title{
Building the Galactic halo from globular clusters: evidence from chemically unusual red giants ${ }^{\star}$
}

\author{
S. L. Martell ${ }^{1}$, J. P. Smolinski ${ }^{2,3}$, T. C. Beers ${ }^{2}$, and E. K. Grebel ${ }^{1}$
}

\author{
1 Astronomisches Rechen-Institut, Zentrum für Astronomie der Universität Heidelberg, 69120 Heidelberg, Germany \\ e-mail: [martell; grebel]@ari.uni-heidelberg.de \\ 2 Department of Physics and Astronomy and JINA (Joint Institute for Nuclear Astrophysics), Michigan State University, \\ East Lansing, MI 48824, USA \\ e-mail: smolin19@msu.edu, beers@pa.msu.edu \\ ${ }^{3}$ Department of Physics and Astronomy, State University of New York College at Oneonta, Oneonta, NY 13820, USA \\ e-mail: smolinjp@oneonta.edu
}

Received 6 July 2011 / Accepted 31 August 2011

\begin{abstract}
We present a spectroscopic search for halo field stars that originally formed in globular clusters. Using moderate-resolution SDSSIII/SEGUE-2 spectra of 561 red giants with typical halo metallicities $(-1.8 \leq[\mathrm{Fe} / \mathrm{H}] \leq-1.0)$, we identify 16 stars, $3 \%$ of the sample, with $\mathrm{CN}$ and $\mathrm{CH}$ bandstrength behavior indicating depleted carbon and enhanced nitrogen abundances relative to the rest of the data set. Since globular clusters are the only environment known in which stars form with this pattern of atypical light-element abundances, we claim that these stars are second-generation globular cluster stars that have been lost to the halo field via normal cluster massloss processes. Extrapolating from theoretical models of two-generation globular cluster formation, this result suggests that globular clusters contributed significant numbers of stars to the construction of the Galactic halo: we calculate that a minimum of $17 \%$ of the present-day mass of the stellar halo was originally formed in globular clusters. The ratio of $\mathrm{CN}$-strong to $\mathrm{CN}$-normal stars drops with Galactocentric distance, suggesting that the inner-halo population may be the primary repository of these stars.
\end{abstract}

Key words. stars: abundances - Galaxy: halo - Galaxy: formation

\section{Introduction}

Light-element abundance inhomogeneities in globular clusters have been a subject of ongoing study for over thirty years. Early observations of a bimodal distribution of $\mathrm{CN}$ bandstrength in globular cluster red-giant (RGB) stars, anticorrelated with $\mathrm{CH}$ bandstrength (e.g., Norris \& Cottrell 1979; Suntzeff 1981; Norris et al. 1984), were interpreted as evidence of anticorrelated ranges of carbon and nitrogen abundances in globular cluster stars (e.g., Bell \& Dickens 1980). Since that time, observations have been extended to include more clusters, fainter stars within individual clusters, and additional elemental abundances. We now know that anticorrelated $\mathrm{C}-\mathrm{N}, \mathrm{O}-\mathrm{Na}$ and $\mathrm{Mg}-\mathrm{Al}$ ranges are present in stars on the red-giant branches of all globular clusters that have been thoroughly studied (e.g., Kayser et al. 2008; Carretta et al. 2009; Smolinski et al. 2011b), and that the C-N anticorrelation is also found among main-sequence stars in several clusters (e.g., Harbeck et al. 2003; Briley et al. 2004; Pancino et al. 2010; Smolinski et al. 2011b). Light-element abundances in globular cluster stars range from scaled-Solar, similar to halo field stars of the same metallicity, to depleted in $\mathrm{C}, \mathrm{O}$ and $\mathrm{Mg}$ and enhanced in N, Na and Al, with between 30\% (Pancino et al. 2010) and $70 \%$ (Carretta et al. 2009) of stars in a given cluster having an atypical abundance pattern.

The atypical abundance pattern resembles the result of hightemperature hydrogen burning: the $\mathrm{CNO}$ cycle converts carbon

* Full Tables 1 and 3 are available in electronic form at the CDS via anonymous ftp to cdsarc.u-strasbg. fr $(130.79 .128 .5)$ or via http://cdsarc.u-strasbg.fr/viz-bin/qcat?]/A+A/534/A136 and oxygen into nitrogen, and the $\mathrm{NeNa}$ and $\mathrm{MgAl}$ cycles increase the abundances of sodium and aluminium while depleting magnesium. The presence of this abundance pattern in old, low-mass main-sequence stars, which have neither the hightemperature fusion zones nor the capacity to transport material between their cores and surfaces, requires that the abundance variations be extrinsic. The fact that the abundance differences between stars with typical and atypical abundance patterns are equally large before and after first dredge-up, which occurs low on the RGB, indicates that the abundance differences are present throughout the stars, and are not merely surface pollution.

As a result, the prevailing model for the origin of primordial light-element abundance variations in globular clusters is that the stars with atypical light-element abundances are a nearly coeval second generation formed from material processed by intermediate- or high-mass stars in the first generation. There are several possible sites for high-temperature fusion processing in the first generation. AGB stars are a common suggestion (e.g., Parmentier et al. 1999) because they have relatively slow winds, they are a site of hot hydrogen burning (e.g., Karakas 2010), and they evolve on timescales of $\sim 10^{8}$ years, quite fast compared to the lifetime of a globular cluster. Other possible sources for high-temperature-processed feedback material are rapidly rotating massive stars (Decressin et al. 2007) and massive binary stars undergoing mass transfer (de Mink et al. 2009), both of which could return more feedback mass in a shorter amount of time than AGB stars, although the stars themselves are less common.

However, all of these possible feedback sources suffer from what has come to be known as the "mass budget problem," the mismatch between the current 1:1 ratio of first- to 
second-generation stars and the concept that the second generation was built entirely from winds from the first generation. Recent theoretical models for globular cluster formation propose two solutions to the mass budget problem: massive first generations and significant gas accretion. The models of Bekki et al. (2007) and D'Ercole et al. (2008) both require a first generation with an initial mass 10 to 20 times its present mass, and have second generations built entirely from AGB winds. In the model of D'Ercole et al. (2008), the second-generation star formation occurs near the center of the cluster, and concludes when type Ia supernovae begin. The Ia $\mathrm{SNe}$ drive an overall expansion of the cluster, causing stars at large radii (primarily first-generation stars) to dissociate from the cluster, thereby reducing the ratio of first- to second-generation stars to present-day levels. AGB stars are also the source of chemical differences between the first and second generation in the model of Conroy \& Spergel (2011), but their model involves a significant amount of gas being accreted from the environment, reducing the mass required for the first generation of stars. The authors investigate the mass-accretion rate for proto-globular clusters in a variety of cosmological environments, and conclude that clusters with initial masses above $10^{4} M_{\odot}$ would have been able to accrete significant amounts of gas in the early Milky Way. They also calculate that the minimum mass for sufficient gas accretion would be lower for globular clusters forming as satellites of dwarf galaxies, a claim that could be tested with observations of intermediate-age star clusters in the Large Magellanic Cloud (LMC). Interestingly, the recent high-resolution study of Mucciarelli et al. (2011) finds no sign of light-element anticorrelations among 14 red giant stars in the massive intermediate-age LMC cluster NGC 1866. The authors suggest that the minimum mass for self-enrichment in the LMC is on the order of $10^{5} M_{\odot}$. Although there are questions about the likelihood that early globular clusters would accrete unprocessed gas from their surroundings with precisely the right metallicity (e.g., Martell 2011), and the gas dynamics involved in mass loss and dilution in globular clusters have been the subject of few advanced numerical studies (e.g., Priestley et al. 2011; D'Ercole et al. 2011), the Conroy \& Spergel (2011) model provides an important discussion of the interplay between early cluster self-enrichment and the galaxy-scale environment.

These scenarios rely implicitly on the high mass and high density of early globular clusters to retain and/or accrete gas well enough to permit a second burst of star formation. It is therefore not surprising that although stars with second-generation-like light-element abundances are found in every globular cluster in the Milky Way, they are not found at all in old open clusters. In studies of individual stars (e.g., Jacobson et al. 2008; Martell \& Smith 2009) and mean abundances (e.g., de Silva et al. 2009) for old open clusters, light-element abundance behavior is found to be distinctly different from what is observed in globular clusters. They are also quite unlikely to have formed in the halo field, but they have recently been found there: Martell \& Grebel (2010) (hereafter MG10) searched the SEGUE survey (Sloan Extension for Galactic Understanding and Exploration, Yanny et al. 2009) for halo giants with unusually strong $\mathrm{UV} /$ blue $\mathrm{CN}$ bands and identified 49 (of roughly 2000) stars likely to have low carbon abundances and high nitrogen abundances. Similarly, Carretta et al. (2010) compiled a sample of 144 metal-poor disk, halo and bulge stars from the literature and identified 2 of those as $\mathrm{Na}$-rich and likely to have originated in globular clusters. In both cases, the authors interpret the field stars with second-generation abundances as stars that formed in globular clusters and were later transferred to the halo through mass-loss processes such as tidal stripping by the Galaxy or two-body interactions within the cluster. It is also claimed in MG10 that their figure of $2.5 \%$ of halo stars having second-generation abundances, seen through the lens of a globular cluster formation model with strong early mass loss (such as, e.g., D'Ercole et al. 2008), implies that as much as $50 \%$ of halo field stars originally formed in globular clusters. Carretta et al. (2010) conclude that early mass loss from globular clusters is "a major building block of the halo", and may also have contributed significantly to the formation of the thick disk. This possibility has been suggested theoretically as well: both Baumgardt et al. (2008) and Marks \& Kroupa (2010) find that early mass loss from globular clusters should contribute significant numbers of stars to the halo field.

In this paper we use data from the SEGUE-2 moderateresolution spectroscopic survey, available as part of the eighth data release of the Sloan Digital Sky Survey (Aihara et al. 2011; Eisenstein et al. 2011; York et al. 2000), to search for CN-strong halo giants like those identified in MG10. This will allow us to make two important extensions to that work: first, by expanding the sample of stars surveyed, we can refine our estimate of $f_{\mathrm{h}}^{2 \mathrm{G}}$, the present-day fraction of $\mathrm{CN}$-strong stars in the halo field; second, we calculate $f_{\mathrm{h}}^{\mathrm{GC}}$, the fraction of halo stars with first- or second-generation chemistry originating in globular clusters, as predicted by several different two-generation cluster formation models.

\section{The data set}

The SDSS and its extensions have acquired ugriz photometry for several hundred million stars. SEGUE, one of three sub-surveys that together formed SDSS-II, extended the ugriz imaging footprint of SDSS-I (Fukugita et al. 1996; Gunn et al. 1998; York et al. 2000; Pier et al. 2003; Gunn et al. 2006; Stoughton et al. 2002; Abazajian et al. 2003, 2004, 2005; Adelman-McCarthy et al. 2006; Adelman-McCarthy et al. 2007, 2008; Abazajian et al. 2009) by approximately $3500 \mathrm{deg}^{2}$, and also obtained $R \simeq 2000$ spectroscopy for approximately 240000 stars over a wavelength range of 3800-9200 A. SEGUE included spectra for a collection of Galactic globular and open clusters, which served as calibrators for the $T_{\text {eff }}, \log (g)$, and $[\mathrm{Fe} / \mathrm{H}]$ scales for all stars observed by SDSS/SEGUE, as processed by the SEGUE Stellar Parameter Pipeline (SSPP; Lee et al. 2008a,b; Allende Prieto et al. 2008b). The SSPP produces estimates of $T_{\text {eff }}, \log (g)$, $[\mathrm{Fe} / \mathrm{H}]$, and radial velocity, along with the equivalent widths and/or line indices for 85 atomic and molecular absorption lines, by processing the calibrated spectra generated by the standard SDSS spectroscopic reduction pipeline (Stoughton et al. 2002). See Lee et al. (2008a) for a detailed discussion of the approaches used by the SSPP; Smolinski et al. (2011a) provides details on the most recent updates to this pipeline, along with additional validations. SEGUE-2 (Rockosi et al., in prep.) expanded the numbers of Galactic stars with available low-resolution SDSS spectra by over 120000 , concentrating in particular on stars with greater distances in order to better sample the outer-halo region of the Galaxy.

As in MG10, SEGUE-2 targets were selected from across a large range in parameter space: we began by selecting all SDSSIII targets from SEGUE-2 plates with $[\mathrm{Fe} / \mathrm{H}] \leq-1.0, \log (g) \leq$ $3.0,(g-r)_{0} \geq 0.2$, and a mean signal-to-noise ratio (SNR) of at least 20 per pixel. Errors on the derived quantities and qualityassurance flags were also required to fall within a certain range: $\sigma_{[\mathrm{Fe} / \mathrm{H}]} \leq 0.5, \sigma_{\log (g)} \leq 0.5, \sigma_{T_{\text {eff }}} \leq 200 \mathrm{~K}$. This initial data set contained 2019 stars. We then identified four stars as carbon-rich using the $\mathrm{C}_{2}$ indices defined in MG10, and removed them from the sample. RGB stars were identified by dividing the data into 
Table 1. Star identifiers, position, photometry and parameters for all program stars from this study and from MG10.

\begin{tabular}{|c|c|c|c|c|c|c|c|c|c|c|c|}
\hline SDSS ID & Plate & MJD & FiberID & $\alpha$ & $\delta$ & $g_{0}$ & $(g-r)_{0}$ & {$[\mathrm{Fe} / \mathrm{H}]$} & $T_{\text {eff }}$ & $\log (g)$ & Survey \\
\hline SDSS J1605+1638 & 3289 & 54910 & 132 & $16: 05: 52.72$ & $16: 38: 59.21$ & 16.04 & 0.663 & -1.07 & 4965 & 2.11 & SEGUE-2 \\
\hline SDSS J0842+2216 & 3373 & 54940 & 51 & 08:42:16.23 & $22: 16: 58.27$ & 16.14 & 0.715 & -1.05 & 4913 & 2.06 & SEGUE-2 \\
\hline SDSS J1528+0759 & 3308 & 54919 & 529 & $15: 28: 51.34$ & 07:59:08.62 & 17.11 & 0.696 & -1.04 & 4950 & 2.29 & SEGUE-2 \\
\hline SDSS J1557+3629 & 3478 & 55008 & 229 & $15: 57: 48.95$ & $36: 29: 33.04$ & 16.77 & 0.735 & -1.03 & 4910 & 2.29 & SEGUE-2 \\
\hline SDSS J1011+1810 & 3178 & 54848 & 269 & 10:11:13.50 & $18: 10: 32.73$ & 17.22 & 0.689 & -1.00 & 4934 & 2.37 & SEGUE-2 \\
\hline SDSS J2243+1322 & 3128 & 54776 & 104 & $22: 43: 31.49$ & $13: 22: 33.68$ & 17.72 & 0.689 & -1.09 & 4877 & 2.55 & SEGUE-2 \\
\hline SDSS J1016+1737 & 3178 & 54848 & 55 & $10: 16: 42.76$ & $17: 37: 23.51$ & 17.44 & 0.619 & -1.05 & 5066 & 2.50 & SEGUE-2 \\
\hline SDSS J1206+3021 & 3181 & 54860 & 73 & 12:06:49.60 & $30: 21: 49.39$ & 18.06 & 0.739 & -1.05 & 4983 & 2.53 & SEGUE-2 \\
\hline SDSS J1210+1042 & 3214 & 54866 & 14 & $12: 10: 11.17$ & $10: 42: 28.64$ & 16.80 & 0.652 & -1.07 & 5025 & 2.56 & SEGUE-2 \\
\hline SDSS J1617+0502 & 3298 & 54924 & 48 & $16: 17: 42.83$ & 05:02:32.70 & 17.54 & 0.663 & -1.06 & 4991 & 2.56 & SEGUE-2 \\
\hline
\end{tabular}

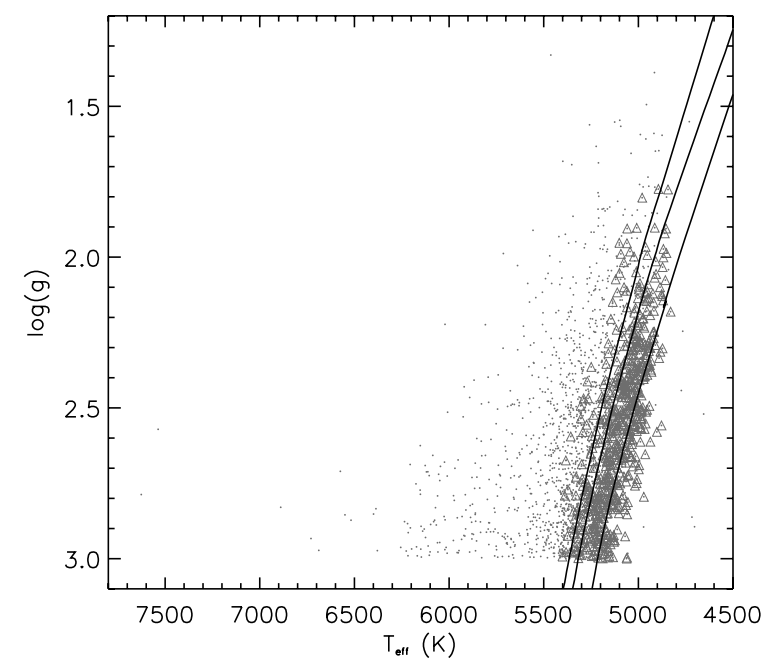

Fig. 1. Surface gravity versus effective temperature for our initial (small gray points) and trimmed (open gray triangles) data sets. Solid black lines are 12 Gyr Dartmouth isochrones with (left to right) $[\mathrm{Fe} / \mathrm{H}]=$ $-1.8,-1.4$, and -1.0 .

0.2-dex-wide metallicity bins and making a recursive 3 -sigma selection about a fiducial sequence in the $\left(\log (g), T_{\text {eff }}\right)$ plane. Since our analysis is based on the $\mathrm{CN}$ and $\mathrm{CH}$ molecular absorption features, which become quite weak at low metallicity, we also restrict our sample to stars with $[\mathrm{Fe} / \mathrm{H}] \geq-1.8$ and a SNR per pixel between $4000 \AA$ and $4100 \AA$ ( $\left(\mathrm{SN}_{\text {blue }}\right)$ above 15 . The RGB, metallicity, and $\mathrm{SN}_{\text {blue }}$ selections together reduce our data set to 561 stars.

Some stars from SEGUE-1 were intentionally re-observed in SEGUE-2, as a way to check for consistency in data reduction and analysis between the two surveys. However, none of the stars in this study were also in the MG10 data set. Table 1 lists SDSS identifiers, the plug plate number, MJD, and fiber number of the SEGUE observation (useful for selecting individual stars from the SDSS Catalog Archive Server ${ }^{1}$ ), right ascension, declination, photometry, SSPP-derived stellar parameters, and survey name (SEGUE-1 or SEGUE-2) for each star in the final trimmed data set and the final data set from MG10. Only the first ten rows of Table 1 are given in the paper, to indicate form and content; a full version is available at the CDS. Figure 1 shows surface gravity versus effective temperature for our initial (small gray points) and trimmed (open gray triangles) data sets, using the $T_{\text {eff }}$ and $\log (g)$ values derived from the spectra by the SSPP. The overplotted solid lines are 12 Gyr Dartmouth isochrones (Dotter et al. 2008) with metallicities of $[\mathrm{Fe} / \mathrm{H}]=-1.8,-1.4$, and -1.0 , spanning the metallicity range of our data set.

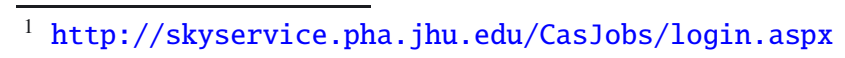

\section{Analysis}

Because we are searching for old, low-mass red giant stars with globular-cluster-like abundance patterns, our analysis follows a process typical in the globular cluster literature. Specifically, we are using index-based techniques developed for globular cluster studies as indicators of carbon and nitrogen abundance, and we infer the presence of the full second-generation light-element abundance pattern from those indices. Overall, we followed the procedure from MG10 fairly closely in analyzing our data, in order for the results to be as compatible as possible.

\subsection{Bandstrength indices}

We use the bandstrength indices $S$ (3839) (Norris et al. 1981) and $S(\mathrm{CH})$ (Martell et al. 2008), which were initially developed for studies of light-element abundance variations in globular clusters, to identify stars with strong $\mathrm{CN}$ bands and weak $\mathrm{CH}$ bands relative to the majority of the halo field. Indices measure the magnitude difference between the integrated flux in the feature (the "science band") and one or two nearby, independent regions of the spectrum (the "sidebands"), in the sense that more absorption in the feature results in a larger index value. Since molecular abundance is strongly controlled by the abundance of the minority species, the $\mathrm{CH}$ band is indicative of $[\mathrm{C} / \mathrm{Fe}]$ while the $\mathrm{CN}$ band traces $[\mathrm{N} / \mathrm{Fe}]$. We are using carbon and nitrogen abundances as an indicator of the "second-generation" abundance pattern of depleted carbon, oxygen, and magnesium and enhanced nitrogen, sodium, and aluminum because that full abundance pattern is consistently found together in Galactic globular clusters.

Figure 2 shows typical spectra from our data set, chosen to illustrate the range of variation in $\mathrm{CN}$ and $\mathrm{CH}$ bandstrength. The upper spectrum (of SDSS J1500+1126) is of a CN-strong star with $T_{\text {eff }}=5250,[\mathrm{Fe} / \mathrm{H}]=-1.70, S(3839)=0.005$ and $S(\mathrm{CH})=0.70$, and the lower spectrum (of SDSS J1524+0550) has similar stellar parameters but relatively weaker $\mathrm{CN}$ and stronger $\mathrm{CH}$ bands $\left(T_{\text {eff }}=5210,[\mathrm{Fe} / \mathrm{H}]=-1.74, S(3839)=\right.$ -0.189 and $S(\mathrm{CH})=0.79)$. The spectra are both normalized near $4500 \AA$ and offset vertically. The shaded areas with lines tipping up to the right mark the sideband (more closely spaced lines) and science band (more broadly spaced lines) of $S$ (3839), and the shaded areas with lines angled down to the right mark the sidebands (more closely spaced lines) and science band (more broadly spaced lines) of $S(\mathrm{CH})$. The bandpasses of both indices are given in Table 2. Errors on the bandstrengths were estimated as in MG10, using a Monte Carlo technique to randomly sample the SEGUE noise vectors and add that noise to the spectra, then measure the standard deviation of the bandstrength over 


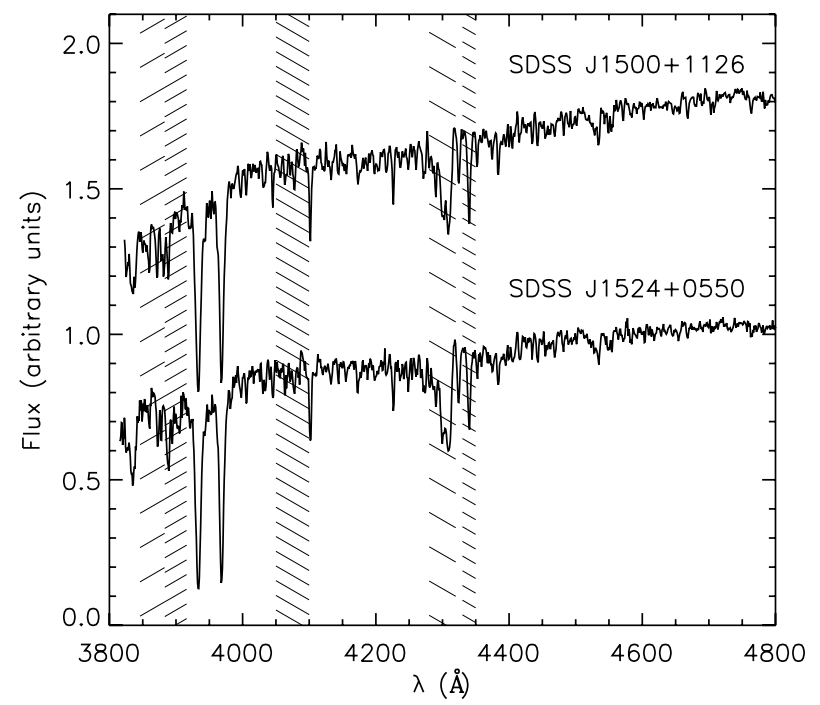

Fig. 2. Two sample spectra from our data set, demonstrating the effects of varying $[\mathrm{C} / \mathrm{Fe}]$ and $[\mathrm{N} / \mathrm{Fe}]$ at fixed stellar parameters. The upper spectrum, of SDSS J1500+1126, is CN-strong while the lower spectrum, of SDSS J1524+0550, is CN-normal. Shaded regions denote the bandpasses of $S(3839)$ and $S(\mathrm{CH})$ : lines angled up to the right mark the sideband (more closely spaced lines) and science band (more broadly spaced lines) of $S$ (3839), and lines angled down to the right mark the sidebands (more closely spaced lines) and science band (more broadly spaced lines) of $S(\mathrm{CH})$.

Table 2. Bandstrength index definitions.

\begin{tabular}{lccc}
\hline \hline Index & Blue sideband & Science band & Red sideband \\
\hline$S(3839)$ & - & $3846-3883 \AA$ & $3883-3916 \AA$ \\
$S(\mathrm{CH})$ & $4050-4100 \AA$ & $4280-4320 \AA$ & $4330-4350 \AA$ \\
\hline
\end{tabular}

100 realizations. Typical values for $\sigma_{S(3839)}$ and $\sigma_{S(C H)}$ are 0.015 and 0.007 , respectively.

\subsection{Identification of $\mathrm{CN}$-Strong candidates}

We identify candidate second-generation halo stars in our data set in two stages, first by selecting those stars with unusually high $S$ (3839) relative to other stars of similar metallicity and evolutionary stage, and then by taking just the subset of those $\mathrm{CN}$-strong stars with appropriate $S(\mathrm{CH})$. Absolute magnitudes for our stars were calculated using a spectroscopic parallax method similar to that used in MG10. We used 12-Gyr Dartmouth isochrones (Dotter et al. 2008), interpolated to the metallicities of our target stars, then use dereddened $(g-r)_{0}$ colors to predict absolute $M_{r}$ magnitudes.

Figure 3 shows $S$ (3839) versus $M_{r}$ for our 561 program stars, divided into 0.1 -dex-wide metallicity bins. The solid lines overplotted are linear fits to the $\mathrm{CN}$-normal stars, and the maximum metallicity in each bin is given in the upper left corner of each panel. The distinctive characteristics of each panel are the dominance of the $\mathrm{CN}$-normal group, the slope of the best-fit line, and the separation of the $\mathrm{CN}$-normal and $\mathrm{CN}$-strong groups. In globular clusters, the fraction of $\mathrm{CN}$-strong stars is roughly $50 \%$ (Kraft 1994), but the slope of the CN bandstrength-luminosity relation and the separation in $\mathrm{CN}$ bandstrength between the two groups behaves similarly to what we observe in field stars. As in MG10, the slope and the separation both decline with decreasing

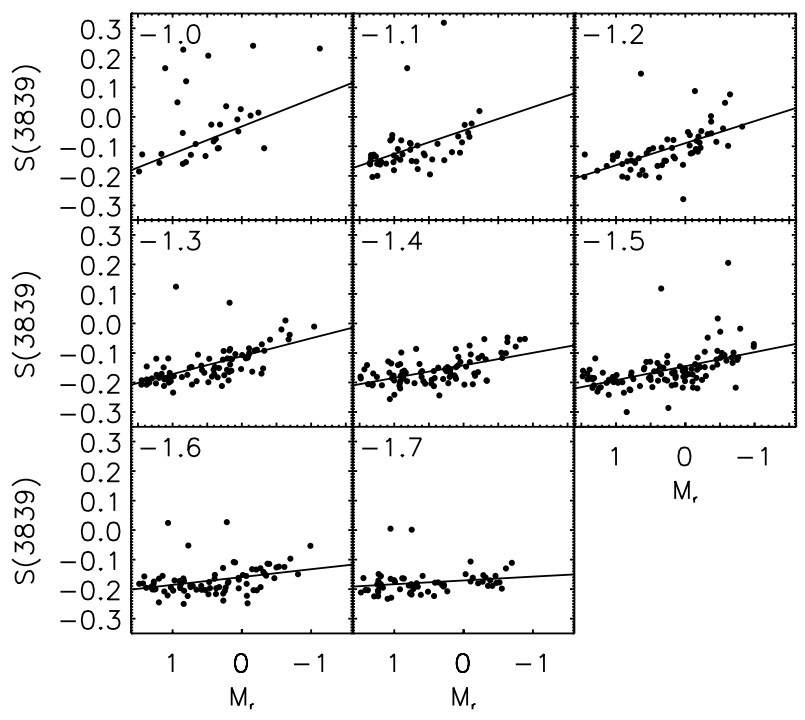

Fig. 3. CN bandstrength index $S$ (3839) versus absolute $r$ magnitude for our full data set, divided into 0.1-dex-wide bins in $[\mathrm{Fe} / \mathrm{H}]$. Maximum $[\mathrm{Fe} / \mathrm{H}]$ in each bin is given in the upper left corner of each panel. Solid lines are best fits to the $\mathrm{CN}$-normal stars in each panel. The slope of the best-fit line and the separation between $\mathrm{CN}$-normal and $\mathrm{CN}$-strong stars both decline with decreasing metallicity.

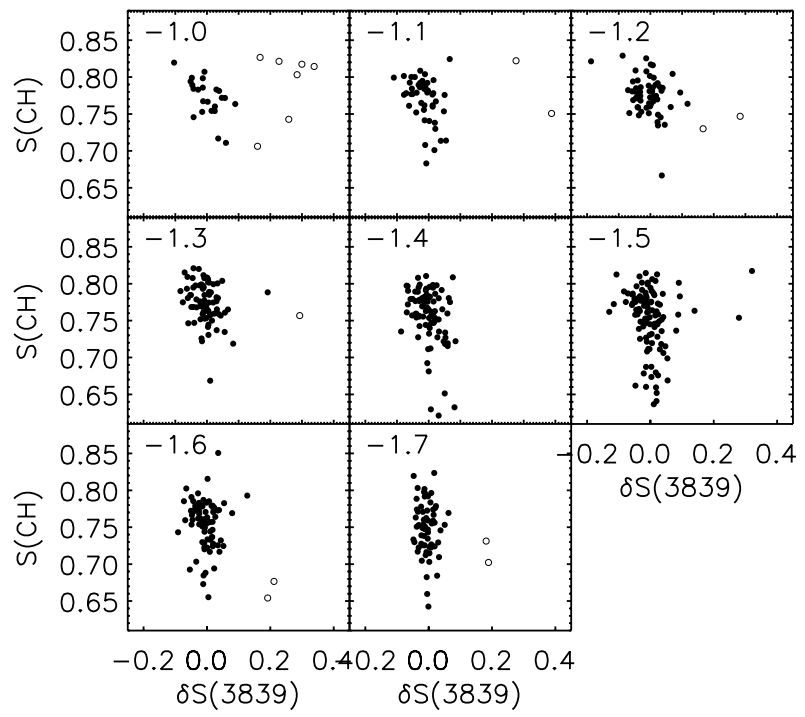

Fig. 4. CN-CH planes for our final data set, divided into 0.1-dex-wide bins in $[\mathrm{Fe} / \mathrm{H}]$. The $\mathrm{CH}$ bandstrength index $S(\mathrm{CH})$ is plotted versus the $\mathrm{CN}$ bandstrength residual $\delta S(3839)$, and candidate globular cluster stars are drawn as open circles. As in Fig. 3, the maximum metallicity in each bin is given in the upper left corner. As is explained in the text, we select as candidate globular cluster stars all those with relatively high $\delta S(3839)$ in the three highest-metallicity bins, and stars with both high $\delta S$ (3839) and $S(\mathrm{CH})$ below the mean $S(\mathrm{CH})$ of the CN-normal group in all other metallicity bins.

metallicity, and the two $\mathrm{CN}$ bandstrength groups become difficult to distinguish at the lowest metallicites.

We measure the quantity $\delta S(3839)$, the difference between an individual star's $\mathrm{CN}$ bandstrength and the best-fit line at the same absolute magnitude, in the plane of Fig. 3. The eight panels of Fig. 4 correspond to the panels of Fig. 3, and show $\delta S$ (3839) versus $\mathrm{S}(\mathrm{CH})$ for each metallicity bin, with candidate secondgeneration field stars plotted as open circles and all other stars plotted as filled circles. Because the $S(\mathrm{CH})$ index has a reduced 
S. L. Martell et al.: Globular cluster stars in the halo

Table 3. Measured and derived parameters for all program stars from this study and from MG10.

\begin{tabular}{cccccccccc}
\hline \hline Plate & MJD & FiberID & $S(3839)$ & $S(\mathrm{CH})$ & CN class & $M_{r}$ & $D(\mathrm{kpc})$ & $R_{\mathrm{GC}}(\mathrm{kpc})$ & Survey \\
\hline 3289 & 54910 & 132 & -0.026 & 0.716 & Normal & 0.31 & 10.3 & 17.7 & SEGUE-2 \\
3373 & 54940 & 51 & 0.004 & 0.760 & Normal & -0.12 & 12.9 & 17.2 & SEGUE-2 \\
3308 & 54919 & 529 & -0.008 & 0.783 & Normal & 0.06 & 18.6 & 25.2 & SEGUE-2 \\
3478 & 55008 & 229 & 0.014 & 0.754 & Normal & -0.23 & 18.0 & 25.5 & SEGUE-2 \\
3178 & 54848 & 269 & 0.035 & 0.763 & Normal & 0.22 & 18.2 & 22.6 & SEGUE-2 \\
3128 & 54776 & 104 & 0.025 & 0.710 & Normal & 0.01 & 25.3 & 30.3 & SEGUE-2 \\
3178 & 54848 & 55 & -0.153 & 0.789 & Normal & 0.80 & 15.9 & 20.5 & SEGUE-2 \\
3181 & 54860 & 73 & -0.106 & 0.819 & Normal & -0.32 & 33.8 & 38.2 & SEGUE-2 \\
3214 & 54866 & 14 & -0.083 & 0.798 & Normal & 0.40 & 14.1 & 19.3 & SEGUE-2 \\
3298 & 54924 & 48 & -0.105 & 0.784 & Normal & 0.33 & 20.4 & 27.6 & SEGUE-2 \\
\hline
\end{tabular}

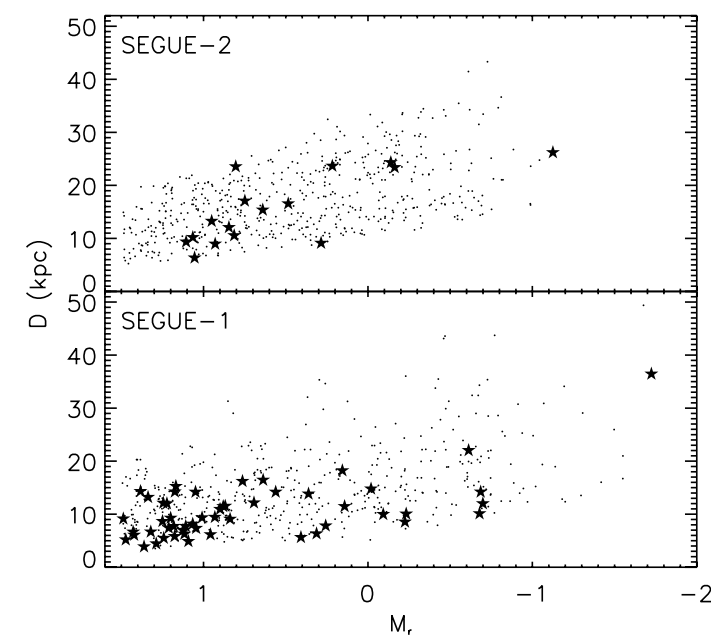

Fig. 5. Heliocentric distance as a function of absolute $r$ magnitude for our final data set (upper panel) and for the MG10 data set (lower panel). $\mathrm{CN}$-normal stars are plotted as small dots, while $\mathrm{CN}$-strong stars are shown as filled stars. Only a randomly selected $25 \%$ of the CN-normal MG10 stars is shown to reduce crowding. In both panels, the lower boundary is set by the bright limit of the SEGUE survey $(g=14)$, and the upper boundary is set by our requirement that $\mathrm{SN}_{\text {blue }}>15$.

sensitivity to carbon abundance at the upper end of our metallicity range, we accept all $\mathrm{CN}$-strong stars with metallicities above $[\mathrm{Fe} / \mathrm{H}]=-1.3$ as candidates, and we select only those $\mathrm{CN}$-strong stars with $\mathrm{S}(\mathrm{CH})$ below the mean of the $\mathrm{CN}$-normal group for stars with metallicity below $[\mathrm{Fe} / \mathrm{H}]=-1.3$. These are the same criteria as were used in MG10, and they return 16 candidate globular cluster stars, $3 \%$ of our sample. This is very similar to the MG10 result based on SEGUE-I stars.

\subsection{Stellar distances}

We calculate heliocentric distances D to our target stars from the distance modulus $\left(r_{0}-M_{r}\right)$. Errors in distance were calculated by randomly sampling the error on $(g-r)_{0}$, then recalculating the distance. The standard deviation in 100 of these realizations was then adopted as $\sigma_{\mathrm{D}}$. One of the goals of the SEGUE-2 survey was to observe stars at greater distances than were observed in SEGUE-1, and while our data set covers a heliocentric distance range very similar to that of MG10, the average star at any fixed $M_{r}$ is more distant for the current data set than for the MG10 data set. The upper panel of Fig. 5 shows our calculated distances versus absolute $M_{r}$ magnitudes for the SEGUE-2 data set, with $\mathrm{CN}$-normal stars drawn as small dots and $\mathrm{CN}$-strong stars drawn as filled stars. The lower panel shows the analogous

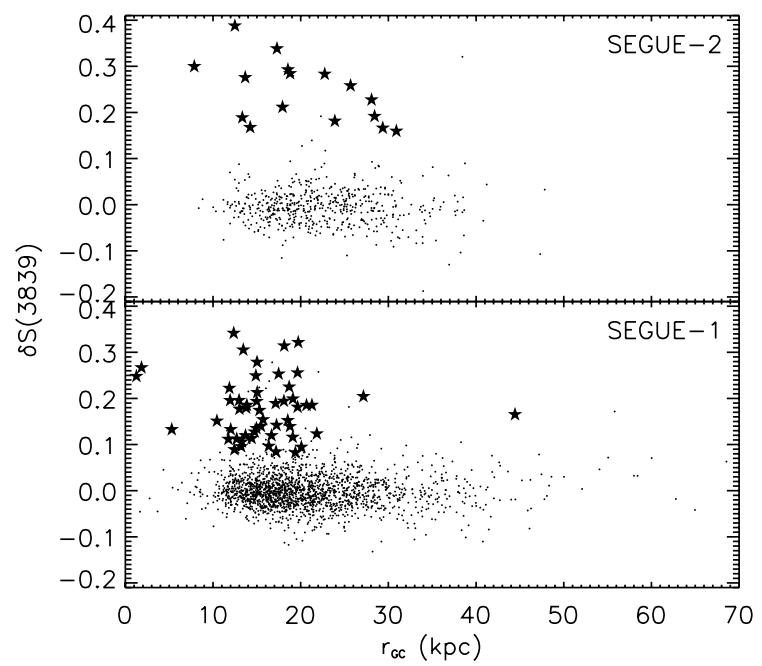

Fig. 6. $\mathrm{CN}$ bandstrength index $\delta S$ (3839) versus Galactocentric distance $r_{\mathrm{GC}}$ for our final data set (upper panel) and the MG10 sample (lower panel). Symbols are the same as in Fig. 5. There is a sharp drop in the number and frequency of $\mathrm{CN}$-strong stars at roughly $20 \mathrm{kpc}$.

data for the MG10 data set, with only a randomly selected $25 \%$ of the MG10 CN-normal stars plotted for visual clarity. In both panels, the stars fill a band that is restricted at small distances by the SEGUE bright limit of $g=14$ and at large distances by our requirement that $\mathrm{SN}_{\text {blue }} \geq 15.0$.

Heliocentric distances are converted geometrically to Galactocentric distances $r_{\mathrm{GC}}$, using the relation $r_{\mathrm{GC}}^{2}=\mathrm{D}^{2}+(8 \mathrm{kpc})^{2}-2 \mathrm{D} \cos (\gamma)$, in which $\gamma$ is the angle on the sky between the Galactic center and the star in question. Table 3 lists plate, MJD, and FiberID identifiers, along with our measurements of $S(3839)$ and $S(C H)$, our determination of a star's CN class (normal or strong), absolute $M_{r}$ magnitude, heliocentric distance, Galactocentric distance, and survey name (SEGUE-1 or SEGUE-2), for the same stars as in Table 1. As with Table 1, only the first ten rows are given in the paper, and a full version of the table is available from CDS. Figure 6 shows $\delta S$ (3839) versus $r_{\mathrm{GC}}$ for our final data set (upper panel) and the MG10 sample (lower panel), using the same symbols as in Fig. 5. The frequency of $\mathrm{CN}$-strong stars appears to drop at roughly $20 \mathrm{kpc}$, with only one $\mathrm{CN}$-strong star beyond $r_{\mathrm{GC}}=30 \mathrm{kpc}$.

To further investigate this result, we visualize the relationship between the frequency of $\mathrm{CN}$-strong stars and Galactocentric distance in two ways: Fig. 7 shows $f_{\mathrm{s}}$, the ratio of $\mathrm{CN}$-strong to $\mathrm{CN}$-normal stars, versus Galactocentric distance for the present data set combined with MG10. Vertical error 


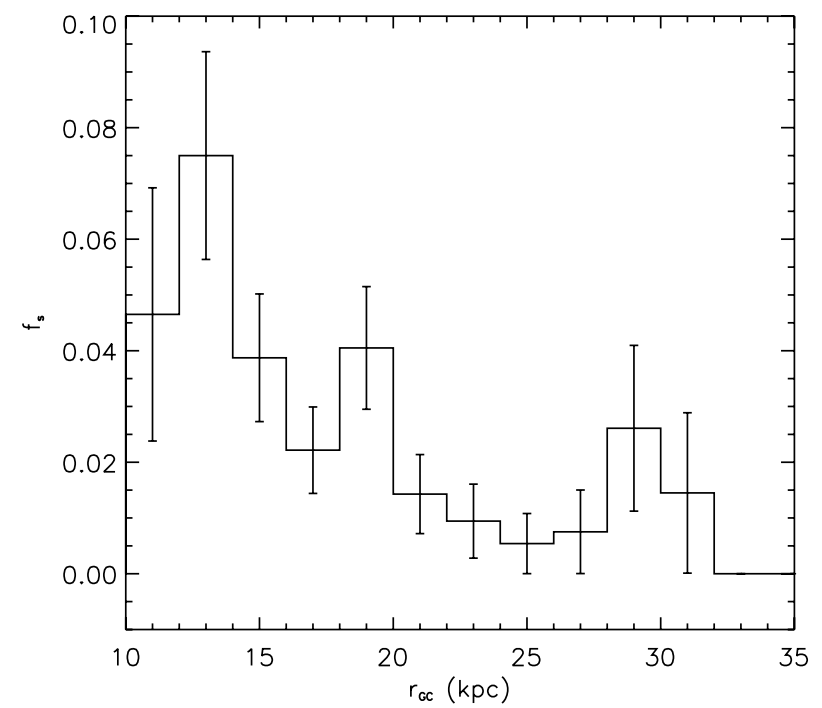

Fig. 7. The ratio of $\mathrm{CN}$-strong to $\mathrm{CN}$-normal stars, $f_{\mathrm{s}}$, as a function of Galactocentric distance for the present data set and the MG10 data combined. Vertical error bars represent the Poisson errors on $f_{\mathrm{s}}$ in each distance bin. The drop in the frequency of $\mathrm{CN}$-strong stars seen in Fig. 5 at $20 \mathrm{kpc}$ is also seen here.

bars represent Poisson errors in $f_{\mathrm{s}}$. The fraction of $\mathrm{CN}$-strong stars is lower at larger $r_{\mathrm{GC}}$, with a transition at $\simeq 20 \mathrm{kpc}$ : relatively small- $r_{\mathrm{GC}}$ stars are consistent with a value for $f_{\mathrm{s}}$ around 0.04 and relatively large- $r_{\mathrm{GC}}$ stars are consistent with a value for $f_{\mathrm{s}}$ around 0.01 . Figure 8 shows cumulative distribution functions of $\delta S$ (3839) for stars with $r_{\mathrm{GC}}<20 \mathrm{kpc}$ (solid line) and $r_{\mathrm{GC}} \geq 20 \mathrm{kpc}$ (dotted line). The cumulative distribution curve for stars with smaller Galactocentric distances climbs continuously for $\delta S(3839)>0.05$, while the curve for more distant stars is flatter in that range, indicating that there are relatively more $\mathrm{CN}$-strong stars at smaller Galactocentric distances. A onesided Kolmogorov-Smirnov test returns a probability of 0.15 that the $\delta S(3839)$ distributions in these two distance ranges were drawn from the same parent population. This result is only a very marginal rejection of the single population hypothesis; however, it clearly points to a reasonable possibility that two parent populations with differing $\mathrm{CN}$-strong fractions exist.

We interpret this drop-off in the frequency of $\mathrm{CN}$-strong stars with Galactocentric distance as a possible sign of a transition from the inner-halo population to the outer-halo population (as discussed by, e.g., Carollo et al. 2007, 2010). Note that this also corresponds to the transition zone reported by de Jong et al. (2010), based on fits to Hess diagrams from the SEGUE vertical photometry stripes. Recent theoretical studies of galaxy formation (e.g., Oser et al. 2010; Font et al. 2011) predict that stars formed in situ are the dominant population at small Galactocentric radii, while stars accreted during minor mergers are the majority at larger Galactocentric distances. Studies of abundances in individual stars in dwarf galaxies do not often report stars with light-element abundances similar to secondgeneration globular cluster stars. In Shetrone et al. (2003) there is only one star out of the 15 surveyed (Fornax 21) with even mildly elevated [Na/Fe], Sadakane et al. (2004) found fairly low $[\mathrm{Na} / \mathrm{Fe}]$ abundances in the three stars they observed in Ursa Minor, and Geisler et al. (2005) find the same result for nine RGB stars in Sculptor. Koch et al. (2008) also report one star in Carina (of eight with measured sodium abundance) with moderate $[\mathrm{Na} / \mathrm{Fe}]$, and Letarte et al. (2010) report one star in Fornax (of 70 with measured sodium abundances) with moderate $[\mathrm{Na} / \mathrm{Fe}]$,

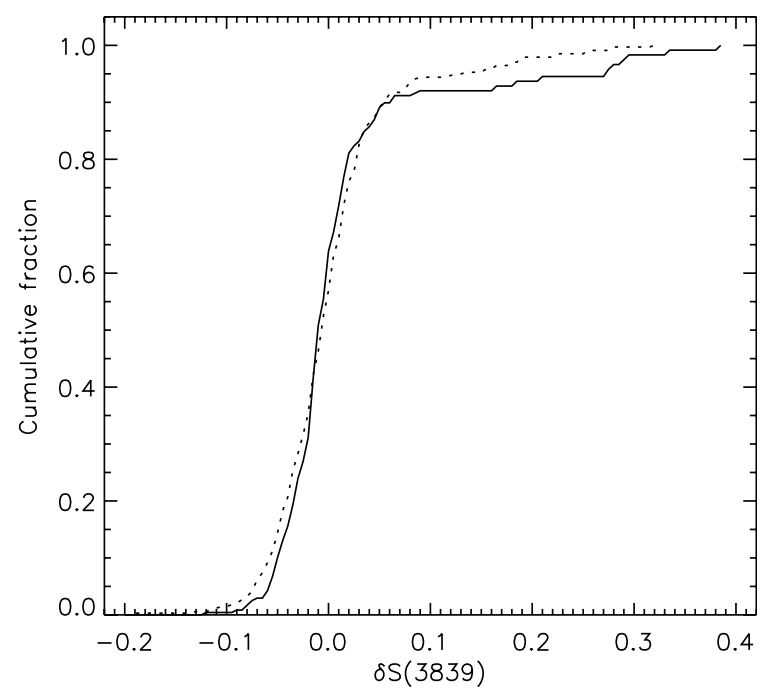

Fig. 8. Cumulative distribution functions for $\delta S$ (3839) for stars with $r_{\mathrm{GC}}<20 \mathrm{kpc}$ (solid line) and $r_{\mathrm{GC}} \geq 20 \mathrm{kpc}$. The fraction of stars rises more quickly at large values of $\delta S$ (3839) for stars with smaller Galactocentric radii, indicating that $\mathrm{CN}$-strong stars are more common in the inner halo.

but neither study finds any stars with the level of sodium abundance enhancement typically found in second-generation globular cluster stars (e.g., Carretta et al. 2009).

A schematic picture of the Galaxy in which the inner halo has significant contributions from globular clusters, while the outer halo is dominated by stars that formed in dwarf galaxies, fits reasonably well with our observation that a higher fraction of stars in the inner halo have light-element abundances similar to those of second-generation globular cluster stars. Spectroscopic surveys currently underway (such as APOGEE; Allende Prieto et al. 2008a), surveys presently being planned as companions to the Gaia mission (e.g., Cacciari 2009), and studies based on nearby stars with accurate proper motions and halo kinematics (such as, e.g., Carollo et al. 2010) will be important ways to explore this question of inner/outer halo origins further: all of them expand the effective search space, either by surveying to larger distances or by focusing on stars with large apogalactic distances.

\section{Interpretation}

The homogeneity of metallicity in present-day globular clusters seems to favor massive models of globular cluster formation, while the observed range in oxygen and sodium abundances (see, e.g., Carretta et al. 2009) indicates that dilution of feedback material and incomplete mixing of the gas that makes up the second stellar generation play a central role in setting final light-element abundances for individual stars. In order to evaluate the veracity of various globular cluster formation models, it is necessary to explore their implications for the formation of the Galactic stellar halo. Massive models, which require a large fraction of first-generation stars to be lost to the halo field at early times, will produce a large enhancement between $f_{\mathrm{h}}^{2 \mathrm{G}}$ and $f_{\mathrm{h}}^{\mathrm{GC}}$.

The recent paper of Schaerer \& Charbonnel (2011) considers this question from a theoretical standpoint, using the globular cluster self-enrichment model of Decressin et al. (2007) to calculate the mass in first-generation stars needed to produce a significant second generation. In the Decressin et al. (2007) model, rapidly rotating massive stars provide chemical 
feedback between the first and second generations, and Schaerer $\&$ Charbonnel (2011) explore the effects of varying the slope of the high-mass end of the stellar initial mass function (IMF) on the resulting second-generation population. Requiring that the present-day ratio of first- to second-generation stars be $1: 2$, the authors calculate that initial globular cluster masses must have been 8 to 10 times their present-day values for a Salpeter IMF. They then compare the mass in first-generation stars that was lost from globular clusters at early times to the mass of the presentday Galactic stellar halo. Since roughly $35 \%$ of the mass in a Salpeter IMF is in low-mass, long-lived stars, and the presentday Galactic globular cluster system comprises $2 \%$ of the mass of the halo, they estimate that escaped first-generation globular cluster stars make up 5-8\% of the mass of the halo.

Schaerer \& Charbonnel (2011) then consider the origin of the second-generation stars found in the halo by MG10 and by Carretta et al. (2010), specifically whether they can reasonably be understood as having been lost to the halo during the strong early mass loss that removed roughly $90 \%$ of the first-generation stars or whether they were transferred during later episodes of globular cluster tidal disruption. Folding in some assumptions involving the initial cluster mass function (ICMF), they conclude that all second-generation stars in the halo were lost at early times. This raises the fraction of cluster mass that was lost in this early phase, which necessarily increases the initial cluster mass to 15 to 25 times the present-day mass and also raises the fraction of halo stars that originally formed in globular clusters to as much as $20 \%$.

However, given the observed examples of globular cluster dissolution in progress, e.g., Palomar 5 (Rockosi et al. 2002; Odenkirchen et al. 2003), NGC 5466 (Belokurov et al. 2006) and the GD-1 stellar stream (Grillmair \& Dionatos 2006), as well as the relative emptiness of the more inhospitable regions of the Gnedin \& Ostriker (1997) "vital diagram", we expect cluster dissolution to be the dominant source of halo field stars with second-generation abundance patterns. Theoretical studies of the ICMF, early star cluster mass loss, and the evolution of the cluster mass function (e.g., Baumgardt et al. 2008; Parmentier et al. 2009; Decressin et al. 2010) all suggest that far more star clusters were initially formed than have survived to the present day, especially at the lower end of the ICMF. The study of Jordi \& Grebel (2010), which used matched-filter techniques to search for faint tidal tails around Galactic globular clusters in the SDSS imaging footprint, found stars with cluster-like photometry outside the tidal radii of a number of clusters without pronounced tidal tails, indicating that stars continue to escape from globular clusters even in the absence of dramatic tidal features. In the limit where cluster dissolution is the sole source of second-generation field stars, we can estimate the number of present-day globular clusters that would need to be disrupted to provide the observed $2.5 \%$ of halo stars with second-generation chemistry, $N_{\mathrm{d}}$. For a typical present-day 1:1 ratio of first- to second-generation stars, and assuming a stellar halo mass of $10^{9} M_{\odot}$ (e.g., Freeman \& Bland-Hawthorn 2002) and a typical present-day globular cluster mass of $5 \times 10^{5} M_{\odot}, N_{\mathrm{d}} \simeq \frac{2 \times f_{\mathrm{h}}^{2 \mathrm{G}} \times M_{\text {halo }}}{\left\langle M_{\mathrm{gcc}}\right\rangle} \simeq 100$. Since the current number of of globular clusters is on the order of 150 , this means that the initial population of globular clusters was significantly larger, but this is not an outrageous claim: Mackey \& van den Bergh (2005) calculate that the ratio between the initial and present-day number of globular clusters in the Galaxy is $3 / 2$, whereas we derive $5 / 3$.

Whatever the details, it seems clear that first-generation stars lost from globular clusters at early times are an important contribution to the construction of the Galactic halo, if twogeneration self-enrichment scenarios for globular cluster formation are indeed the correct model. The models of Decressin et al. (2007), D'Ercole et al. (2008), Carretta et al. (2010), and Vesperini et al. (2010) all predict that $90 \%$ of the first generation ought to be lost from globular clusters at early times, while Conroy (2011) estimates that as much as $95 \%$ of the first generation ought to be lost. The mass of first-generation stars lost during this phase by the $N_{\mathrm{GC}}$ globular clusters in the present-day system can be expressed as $M_{1 \mathrm{G}}^{\text {lost }}=M_{\mathrm{GC} \text { system }} \times \frac{1}{2} \times\left(\frac{1}{1-f_{\text {lost }}}-1\right)$. Considering the early contributions of first-generation stars from globular clusters that have survived to the present day as well as the clusters that have completely dissolved, we estimate that $f_{\mathrm{h}}^{\mathrm{GC}}$ has a minimum value of $\frac{M_{1 \mathrm{G}}^{\text {lost }}}{M_{\text {halo }}}+\left(\frac{N_{\mathrm{d}}}{N_{\mathrm{GC}}} \times \frac{M_{1 \mathrm{G}}^{\text {lost }}}{M_{\text {halo }}}\right)+M_{\mathrm{GC} \text { system }}=$ $2 \% \times\left(\frac{9}{2}+\frac{12}{3}\right)=17 \%$ of halo field stars, with both first- and second-generation abundance patterns, originally formed within globular clusters, plus an additional unknown contribution of first-generation stars from clusters too low-mass to self-enrich that have dispersed by the present day.

\section{Future challenges}

Based on the current two-generation models for globular cluster formation, the early phase of globular cluster formation must have contributed a significant number of first-generation stars to the Galactic halo. From the data presented herein, by MG10, and by Carretta et al. (2010), it is also clear that second-generation globular cluster stars are a component of the halo field. Further investigations of the process of globular cluster formation will help to clarify the role that globular clusters played in the early assembly of the Galactic halo. There are two specific directions that new observations could take that would be particularly insightful: investigating the relationship between the minimum mass for cluster self-enrichment and the larger galactic environment, and searching for star clusters still in the process of selfenrichment.

These studies will necessarily require observations of star clusters outside the Milky Way, as a way to compare cluster formation in different large-scale environments. Observing very young star clusters may offer the opportunity to see the process of self-enrichment in progress. Carretta et al. (2010) suggest that clusters in the process of violent relaxation (at a few $\times 10^{7}$ years old) should have a central compact cluster, an extended, nonbound halo and outflowing gas. It is suggested in Conroy (2011) that the extended clusters discovered in M 31 by Huxor et al. (2005) are in this state (see also Huxor et al. 2011). If this is the case, the stars at large radius in the extended clusters should have almost entirely first-generation chemistry, and strong radial gradients in light-element abundances, most readily observable in $\mathrm{CN}$ and $\mathrm{CH}$ molecular features, should be present.

To explore the question of the minimum cluster mass for self-enrichment, it would be useful to consider star clusters in lower-mass galaxies. Conroy \& Spergel (2011) claim that the weaker tidal field of the Large Magellanic Cloud (LMC) ought to permit the formation of a second stellar generation at lower cluster mass than in the Milky Way. Spectroscopic observations of red giant-branch stars in intermediate-age populous clusters in the LMC would be particularly interesting because recent photometric work (e.g., Mackey et al. 2008; Milone et al. 2009; Goudfrooij et al. 2011) has uncovered broadened or split main-sequence turnoffs in a large fraction of them. Since they are much younger than Galactic globular clusters (typically 1-4 Gyr), age differences on the order of a few hundred million 
years are visible at the turnoff, and this age difference is suggestively similar to the age difference expected between first- and second-generation stars in Galactic globular clusters. Obtaining spectra of turnoff stars at the distance of the LMC would be extremely difficult - indeed, high-resolution spectroscopic studies of RGB stars in these clusters are limited to fairly small samples (e.g., Mucciarelli et al. 2008). However, a lower-resolution study using a multiobject spectrograph could collect $\mathrm{CN}$ and $\mathrm{CH}$ data analogous to the data presented in this paper, and would allow a search for star-to-star variations in carbon and nitrogen abundance for a larger data set per cluster. A modified version of the SSPP has been developed for application to spectra with resolving power as low as $R \sim 1000$ ), and is already being used with a variety of low-resolution data from non-SDSS sources (e.g., see Li et al. 2010; Humphreys et al. 2011). These data would also permit an investigation of theoretical claims (e.g., Conroy \& Spergel 2011) that lower-mass galaxies permit the formation of multiple stellar generations in lower-mass star clusters, by comparing the $\mathrm{CN}-\mathrm{CH}$ behavior of intermediate-age LMC clusters across a range of masses.

Acknowledgements. S.L.M. was supported by Sonderforschungsbereich SFB 881 "The Milky Way System" (subprojects A2 and A5) of the German Research Foundation (DFG). JPS and TCB acknowledge partial funding of this work from grants PHY 02-1783 and PHY 08-22648: Physics Frontiers Center/Joint Institute for Nuclear Astrophysics (JINA), awarded by the National Science Foundation.

Funding for SDSS-III has been provided by the Alfred P. Sloan Foundation, the Participating Institutions, the National Science Foundation, and the US Department of Energy. The SDSS-III web site is http://www. sdss3.org/. SDSS-III is managed by the Astrophysical Research Consortium for the Participating Institutions of the SDSS-III Collaboration including the University of Arizona, the Brazilian Participation Group, Brookhaven National Laboratory, University of Cambridge, University of Florida, the French Participation Group, the German Participation Group, the Instituto de Astrofisica de Canarias, the Michigan State/Notre Dame/JINA Participation Group, Johns Hopkins University, Lawrence Berkeley National Laboratory, Max Planck Institute for Astrophysics, New Mexico State University, New York University, Ohio State University, Pennsylvania State University, University of Portsmouth, Princeton University, the Spanish Participation Group, University of Tokyo, University of Utah, Vanderbilt University, University of Virginia, University of Washington, and Yale University.

\section{References}

Abazajian, K., Adelman-McCarthy, J. K., Agüeros, M. A., et al. 2003, AJ, 126, 2081

Abazajian, K., Adelman-McCarthy, J. K., Agüeros, M. A., et al. 2004, AJ, 128, 502

Abazajian, K., Adelman-McCarthy, J. K., Agüeros, M. A., et al. 2005, AJ, 129, 1755

Abazajian, K. N., Adelman-McCarthy, J. K., Agüeros, M. A., et al. 2009, ApJS, 182,543

Adelman-McCarthy, J. K., Agüeros, M. A., Allam, S. S., et al. 2006, ApJS, 162, 38

Adelman-McCarthy, J. K., Agüeros, M. A., Allam, S. S., et al. 2007, ApJS, 172, 634

Adelman-McCarthy, J. K., Agüeros, M. A., Allam, S. S., et al. 2008, ApJS, 175, 297

Aihara, H., Allende Prieto, C., An, D., et al. 2011, ApJS, 193, 29

Allende Prieto, C., Majewski, S. R., Schiavon, R., et al. 2008a, Astron. Nachr., 329, 1018

Allende Prieto, C., Sivarani, T., Beers, T. C., et al. 2008b, AJ, 136, 2070

Baumgardt, H., Kroupa, P., \& Parmentier, G. 2008, MNRAS, 384, 1231

Bekki, K., Campbell, S. W., Lattanzio, J. C., \& Norris, J. E. 2007, MNRAS, 377, 335

Bell, R. A., \& Dickens, R. J. 1980, ApJ, 242, 657

Belokurov, V., Evans, N. W., Irwin, M. J., Hewett, P. C., \& Wilkinson, M. I. 2006, ApJ, 637, L29

Briley, M. M., Cohen, J. G., \& Stetson, P. B. 2004, AJ, 127, 1579

Cacciari, C. 2009, Mem. Soc. Astron. Ital., 80, 97

Carollo, D., Beers, T. C., Lee, Y. S., et al. 2007, Nature, 450, 1020

Carollo, D., Beers, T. C., Chiba, M., et al. 2010, ApJ, 712, 692
Carretta, E., Bragaglia, A., Gratton, R. G., et al. 2009, A\&A, 505, 117 Carretta, E., Bragaglia, A., Gratton, R. G., et al. 2010, A\&A, 516, A55 Conroy, C. 2011, ApJ, submitted [arXiv: 1101.2208]

Conroy, C., \& Spergel, D. N. 2011, ApJ, 726, 36

de Jong, J. T. A., Yanny, B., Rix, H.-W., et al. 2010, ApJ, 714, 663

de Mink, S. E., Pols, O. R., Langer, N., \& Izzard, R. G. 2009, A\&A, 507, L1

de Silva, G. M., Gibson, B. K., Lattanzio, J., \& Asplund, M. 2009, A\&A, 500, L25

Decressin, T., Meynet, G., Charbonnel, C., Prantzos, N., \& Ekström, S. 2007, A\&A, 464, 1029

Decressin, T., Baumgardt, H., Charbonnel, C., \& Kroupa, P. 2010, A\&A, 516, A73

D’Ercole, A., Vesperini, E., D’Antona, F., McMillan, S. L. W., \& Recchi, S. 2008, MNRAS, 391, 825

D’Ercole, A., D'Antona, F., \& Vesperini, E. 2011, MNRAS, 415, 1304

Dotter, A., Chaboyer, B., Jevremović, D., et al. 2008, ApJS, 178, 89

Eisenstein, D. J., Weinberg, D. H., Agol, E., et al. 2011, AJ, 142, 72

Font, A. S., McCarthy, I. G., Crain, R. A., et al. 2011, MNRAS, 1162

Freeman, K., \& Bland-Hawthorn, J. 2002, ARA\&A, 40, 487

Fukugita, M., Ichikawa, T., Gunn, J. E., et al. 1996, AJ, 111, 1748

Geisler, D., Smith, V. V., Wallerstein, G., Gonzalez, G., \& Charbonnel, C. 2005 , AJ, 129, 1428

Gnedin, O. Y., \& Ostriker, J. P. 1997, ApJ, 474, 223

Goudfrooij, P., Puzia, T. H., Chandar, R., \& Kozhurina-Platais, V. 2011, ApJ, 737,4

Grillmair, C. J., \& Dionatos, O. 2006, ApJ, 643, L17

Gunn, J. E., Carr, M., Rockosi, C., et al. 1998, AJ, 116, 3040

Gunn, J. E., Siegmund, W. A., Mannery, E. J., et al. 2006, AJ, 131, 2332

Harbeck, D., Smith, G. H., \& Grebel, E. K. 2003, AJ, 125, 197

Humphreys, R. M., Beers, T. C., Cabanela, J. E., et al. 2011, AJ, 141, 131

Huxor, A. P., Tanvir, N. R., Irwin, M. J., et al. 2005, MNRAS, 360, 1007

Huxor, A. P., Ferguson, A. M. N., Tanvir, N. R., et al. 2011, MNRAS, 414, 770

Jacobson, H. R., Friel, E. D., \& Pilachowski, C. A. 2008, AJ, 135, 2341

Jordi, K., \& Grebel, E. K. 2010, A\&A, 522, A71

Karakas, A. I. 2010, MNRAS, 403, 1413

Kayser, A., Hilker, M., Grebel, E. K., \& Willemsen, P. G. 2008, A\&A, 486, 437

Koch, A., Grebel, E. K., Gilmore, G. F., et al. 2008, AJ, 135, 1580

Kraft, R. P. 1994, PASP, 106, 553

Lee, Y. S., Beers, T. C., Sivarani, T., et al. 2008a, AJ, 136, 2022

Lee, Y. S., Beers, T. C., Sivarani, T., et al. 2008b, AJ, 136, 2050

Letarte, B., Hill, V., Tolstoy, E., et al. 2010, A\&A, 523, A17

Li, H. N., Christlieb, N., Schörck, T., et al. 2010, A\&A, 521, A10

Mackey, A. D., \& van den Bergh, S. 2005, MNRAS, 360, 631

Mackey, A. D., Broby Nielsen, P., Ferguson, A. M. N., \& Richardson, J. C. 2008, ApJ, 681, L17

Marks, M., \& Kroupa, P. 2010, MNRAS, 406, 2000

Martell, S. L. 2011, Astron. Nachr., 332, 467

Martell, S. L., \& Grebel, E. K. 2010, A\&A, 519, A14

Martell, S. L., \& Smith, G. H. 2009, PASP, 121, 577

Martell, S. L., Smith, G. H., \& Briley, M. M. 2008, PASP, 120, 7

Milone, A. P., Bedin, L. R., Piotto, G., \& Anderson, J. 2009, A\&A, 497, 755

Mucciarelli, A., Carretta, E., Origlia, L., \& Ferraro, F. R. 2008, AJ, 136, 375

Mucciarelli, A., Cristallo, S., Brocato, E., et al. 2011, MNRAS, 413, 837

Norris, J., \& Cottrell, P. L. 1979, ApJ, 229, L69

Norris, J., Cottrell, P. L., Freeman, K. C., \& Da Costa, G. S. 1981, ApJ, 244, 205

Norris, J., Freeman, K. C., \& Da Costa, G. S. 1984, ApJ, 277, 615

Odenkirchen, M., Grebel, E. K., Dehnen, W., et al. 2003, AJ, 126, 2385

Oser, L., Ostriker, J. P., Naab, T., Johansson, P. H., \& Burkert, A. 2010, ApJ, 725,2312

Pancino, E., Rejkuba, M., Zoccali, M., \& Carrera, R. 2010, A\&A, 524, A44

Parmentier, G., Jehin, E., Magain, P., et al. 1999, A\&A, 352, 138

Parmentier, G., Goodwin, S. P., Kroupa, P., \& Baumgardt, H. 2009, Ap\&SS, 324, 327

Pier, J. R., Munn, J. A., Hindsley, R. B., et al. 2003, AJ, 125, 1559

Priestley, W., Ruffert, M., \& Salaris, M. 2011, MNRAS, 411, 1935

Rockosi, C. M., Odenkirchen, M., Grebel, E. K., et al. 2002, AJ, 124, 349

Sadakane, K., Arimoto, N., Ikuta, C., et al. 2004, PASJ, 56, 1041

Schaerer, D., \& Charbonnel, C. 2011, MNRAS, 413, 2297

Shetrone, M., Venn, K. A., Tolstoy, E., et al. 2003, AJ, 125, 684

Smolinski, J. P., Lee, Y. S., Beers, T. C., et al. 2011a, AJ, 141, 89

Smolinski, J. P., Martell, S. L., Beers, T. C., \& Lee, Y. S. 2011b, AJ, 142, 126

Stoughton, C., Lupton, R. H., Bernardi, M., et al. 2002, AJ, 123, 485

Suntzeff, N. B. 1981, ApJS, 47, 1

Vesperini, E., McMillan, S. L. W., D’Antona, F., \& D’Ercole, A. 2010, ApJ, 718, L112

Yanny, B., Rockosi, C., Newberg, H. J., et al. 2009, AJ, 137, 4377

York, D. G., Adelman, J., Anderson, Jr., J. E., et al. 2000, AJ, 120, 1579 